\title{
Revisión sobre los conocimientos actuales de dehiscencia del canal semicircular posterior
}

\author{
Review of current knowledge of the dehiscence \\ in the semicircular posterior canal
}

Raúl Lorenzo L', Ana Isabel Cisneros G', Jaime Whyte $\mathbf{0}^{1}$.

\begin{abstract}
RESUMEN
La dehiscencia del canal semicircular posterior es una patología rara y con baja incidencia, por ello hemos realizado una revisión de los conocimientos actuales de esta entidad.

Se ha realizado una búsqueda bibliográfica desde 1998 hasta diciembre de 2016 de toda la literatura publicada sobre la misma en las bases de datos Allied and Complementary Medicine Database and the Embase, Health Management Information Consortium, Scopus, Consortium, Medline, PsycINFO y Scielo.

Se han encontrado y revisado 53 trabajos relacionados con el tema.

La dehiscencia del canal semicircular posterior presenta una prevalencia variable; 0,3\%-4,5\% en adultos y 1,2\%-20\% en niños. Su localización puede ser hacia el golfo de la yugular o fosa cerebral posterior. Los pacientes pueden ser asintomáticos o presentar clínica auditiva y/o vestibular. La tomografía computarizada y la prueba de potenciales vestibulares miogénicos evocados permiten establecer el diagnóstico de certeza. En el tratamiento quirúrgico la vía de abordaje de elección es la transmastoidea y las técnicas del cierre del canal son el "plugging" y el "resurfacing".
\end{abstract}

Palabras clave: Dehiscencia, canal semicircular posterior, hueso temporal humano.

\begin{abstract}
The posterior semicircular canal dehiscence is a rare pathology and it has a low incidence. We have realized a review about the current knowledge of this entity.

We have performed a bibliographic research from 1998 to 2016 December about the literature published in this subject, in the data basis Allied and Complementary Medicine Database and the Embase, Health Management Information Consortium, Scopus, Consortium, Medline, PsycINFO y Scielo.

I thas been found and reviewed 53 papers about the topic.

The posterior semicircular canal dehiscence has a variable prevalence: 0,3\%-4-5\% in adults and 1,2\%-20\% in children. The location can be in the jugular bulb or in the posterior brain fossa. Some patients can be asymptomatic, whereas others can have
\end{abstract}

1 Departamento de Anatomía e Histología Humanas, Universidad de Zaragoza.

Recibido el 21 de diciembre, 2016. Aceptado el 11 de enero, 2017. 
auditory and/or vestibular signs and symptoms. Computed tomography and test of vestibular evoked myogenic potentials allow the diagnosis of certainty. In the surgical treatment the approach of choice is transmastoid and techniques to close the canal are plugging and resurfacing.

Key words: Dehiscence, posterior semicircular canal, human temporal bone.

\section{INTRODUCCIÓN}

La dehiscencia del canal semicircular posterior es una forma de vestibulopatía periférica que se define como la ausencia de cobertura ósea en la cubierta del propio canal hacia la fosa cerebral posterior 0 hacia el golfo de la vena yugular. Minor ${ }^{1}$ describió en 1998 los primeros casos de dehiscencia del canal semicircular superior y tuvieron que trascurrir cinco años para que Krombach ${ }^{2}$ notificara los primeros casos de la dehiscencia del canal semicircular posterior.

La dehiscencia del canal semicircular posterior es una patología rara, muy poco prevalente y de difícil sospecha dado que los pacientes pueden ser asintomáticos o presentar clínica auditiva y vestibular variable, la cual puede simular otras patologías auditivas.

El objetivo de este trabajo es realizar una revisión bibliográfica sobres los conocimientos actuales de esta patología.

Se ha realizado una búsqueda bibliográfica desde marzo del año 1998 hasta diciembre del año 2016 de todos los conocimientos publicados sobre la dehiscencia del canal semicircular posterior en las bases de datos Allied and Complementary Medicine Database and the Embase, Health Management Information Consortium, Scopus, Consortium, Medline, PsycINFO y Scielo, introduciendo las siguientes palabras claves (key words): "semicircular canal dehiscence"; "semicircular canal dehiscence syndrome"; "posterior semicircular canal dehiscence"; "bilateral posterior semicircular canal dehiscence"; "clínical manifestations of posterior semicircular canal dehiscence"; "operative management of posterior semicircular canal dehiscence" obteniendo 477, 206, 83, 19, 2, 2 artículos respectivamente. Una vez revisados, 53 hacían referencia a la dehiscencia del canal semicircular posterior. Estos trabajos incluían estudios analíticos observacionales prospectivos y retrospectivos, estudios descriptivos transversales, metaanálisis, presentaciones de casos y estudios analíticos cuasi experimentales antes y después.

\section{ETIOLOGÍA}

La génesis de la dehiscencia del canal semicircular posterior no está claramente definida. En la actualidad se han descrito dos teorías: congénita y adquirida.

Origen congénito: Se explica por la detención o retraso de la osificación sobre la cubierta del canal durante su desarrollo. A favor de este origen Whyte y $\mathrm{col}^{3}$ notificaron el hallazgo de una dehiscencia del canal semicircular posterior en un feto de 32 semanas, fecha en que el canal debería haber completado su osificación y Hagiwara y $\mathrm{col}^{4}$ obtuvieron diferencias significativas al evaluar la incidencia de dehiscencia de los canales semicirculares verticales en niños y en adultos; $27,3 \%$ frente al 2,5\% respectivamente. Apoyando este origen, se han comunicado alteraciones congénitas que asocian dehiscencia del canal semicircular posterior a distintos síndromes, como son los síndromes de Alagille y síndrome de Waardenburg ${ }^{5}$, síndrome de Apert ${ }^{6}$, síndrome de Hallemann-Streiff ${ }^{7}$, malformaciones Chiari tipo $I^{8} \mathrm{y}$ síndrome de Usher ${ }^{9}$.

Origen adquirido: Para explicar esta teoría se ha formulado la hipótesis de que en los casos de una anormalmente fina capa de hueso cubriendo el canal semicircular posterior un segundo evento ocasionaría la rotura del mismo. Cisneros y collo concluyeron que patrones radiológicos de canales semicirculares posterior con espesor adelgazado al sufrir un evento pueden evolucionar a patrones "dehiscentes" y Chen y col (2009)11 propusieron que los canales semicirculares posteriores tienen una mayor predisposición de presentar un adelgazamiento (o dehiscencia) en el desarrollo 
porque están más cerca de los senos venosos durales de la fosa cerebral posterior. Como causas de este segundo evento también se han descrito algunas patologías, entre las cuales destacan la presencia de colesteatoma en el ápex de la porción petrosa del hueso temporal ${ }^{12}$, la displasia fibrosa ${ }^{13}$, la histiocitosis del hueso temporal, el granuloma eosinofílico y la cirugía del saco endolinfático ${ }^{14}$. Gopen y $\mathrm{col}^{6}$ describieron dos dehiscencias posquirúrgicas; una tras una cirugía por un schwannoma y otra, tras una mastoidectomía por otomastoiditis crónica, y Cremer y col ${ }^{15}$ una fístula iatrogénica posoperatoria.

\section{Localización}

Dos son las localizaciones anatómicas relacionadas con la ausencia de cobertura ósea en el canal semicircular posterior: el golfo de la vena yugular interna $^{16-19}$ y la fosa cerebral posterior ${ }^{20,21}$.

Los trabajos de Krombach ${ }^{2}$ y Erdogan ${ }^{22}$ sitúan la dehiscencia en la cubierta externa, Brantberg ${ }^{12}$ en la ampolla del canal, Gopen ${ }^{6}$ en las porciones cefálicas 0 caudales y Dang ${ }^{23}$ en la rama común.

\section{Estructura}

Friedmann y $\mathrm{col}^{24}$ describen un caso de un canal posterior erosionado por el golfo de la vena yugular. En el canal no se encontraron procesos inflamatorios, fibróticos, reactivos, ni actividad resortiva en los bordes de la dehiscencia y Whyte y $\mathrm{col}^{3}$ analizaron una dehiscencia del canal semicircular posterior en un feto de 32 semanas cuyos bordes óseos eran lisos y no presentaban actividad osteoclástica. Nomiya y $\mathrm{col}^{25}$ observaron dos huesos que presentaban microfracturas.

\section{Morfología y longitud}

Krombach y $\mathrm{col}^{2}$ obtuvieron una longitud media de 2,3 \pm 0,2 $\mathrm{mm}$ (rango de 2-4 mm), Friedmann y $\mathrm{col}^{16}$ comunicaron una longitud de $0,6 \mathrm{~mm}$ (diámetro anteroposterior) y $1,0 \mathrm{~mm}$ (diámetro supero-inferior), Whyte ${ }^{3}$ describió una dehiscencia de forma elíptica cuyas dimensiones fueron de 3,4 $\mathrm{mm}$ de largo x 0,67 mm de ancho en la zona más superior de la elipse $\times 1,42 \mathrm{~mm}$ de ancho en la zona más inferior. Las medidas de la dehiscencia halladas por Dang ${ }^{23}$ en la rama común fueron de 2-3 mm y por último Saxby y $\mathrm{co}^{21}$ obtuvieron una longitud media de 1,7 $\mathrm{mm}$ con un rango de 1,2$2,5 \mathrm{~mm}$.

\section{PREVALENCIA}

La prevalencia radiológica de esta patología en adultos es variable y baja, dado que tiene un rango que oscila entre 4,5\%-0,3\% (Tabla 1).

Esta variabilidad pensamos que se debe a diferentes factores, como son: el grosor de corte utilizado en la tomografía computarizada, el tamaño de la muestra del estudio, la obtención de resultados unas veces por pacientes y otras por hueso temporal y la falta de acuerdo para definir

Tabla 1. Prevalencia de la dehiscencia del canal semicircular posterior en adultos

\begin{tabular}{|lccccc|}
\hline & $\begin{array}{c}\text { Tamaño muestral } \\
\text { (pacientes) }\end{array}$ & $\begin{array}{c}\text { Pacientes } \\
\text { con dehiscencia }\end{array}$ & $\begin{array}{c}\text { Prevalencia } \\
\text { por paciente }\end{array}$ & $\begin{array}{c}\text { Prevalencia } \\
\text { por hueso } \\
\text { temporal }\end{array}$ & $\begin{array}{c}\text { Grosor del } \\
\text { corte (TC) }\end{array}$ \\
\hline Krombach et al (2003) & 507 & 23 & $4,5 \%$ & $3,6 \%$ & $1,0 \mathrm{~mm}$ \\
Zhou et al (2007) & 65 & 1 & $1,5 \%$ & $0,7 \%$ & - \\
Crovetto et al (2010) & 318 & 2 & $0,6 \%$ & $0,3 \%$ & $0,5 \mathrm{~mm}$ \\
Erdogan et al (2011) & 410 & 5 & $1,2 \%$ & $0,98 \%$ & $0,3 \mathrm{~mm}$ \\
Stimmer et al (2012) & 350 & 8 & $2,3 \%$ & $1,2 \%$ & $1,0 \mathrm{~mm}$ \\
Elmali et al (2013) & 850 & 13 & $1,5 \%$ & 0,76 & $1,0 \mathrm{~mm}$ \\
Russo et al (2014) & 412 & 5 & $1,2 \%$ & $1,2 \%$ & $0,625 \mathrm{~mm}$ \\
Cisneros et al (2014) & 318 & 2 & $0,6 \%$ & $0,3 \%$ & $0,65 \mathrm{~mm}$ \\
\hline
\end{tabular}


una dehiscencia radiológica, haciendo referencia a los casos dudosos denominados "casi dehiscentes" y que podían ser debidos a una pared fina 0 cartilaginosa que no se aprecia con TC.

La prevalencia en niños es mayor (Tabla 2). Nótese en la tabla que conforme aumenta la edad, disminuye la prevalencia de dehiscencia.

Pensamos al igual que Hawigara ${ }^{4}$ que la tomografía computarizada no permite la visualización del hueso inmaduro, este hecho justificaría el aumento de prevalencia en pacientes de edad pediátrica.

\section{Comparación de la prevalencia en niños y adultos}

Hagiwara y $\mathrm{col}^{4}$ compararon la prevalencia de dehiscencias radiológicas de los canales semicirculares verticales (superior y posterior) en niños y en adultos obteniendo diferencias significativas (27,3\%-2,5\% respectivamente).

\section{Distribución por sexo y predisposición del canal}

La dehiscencia del canal semicircular posterior tiene un predominio en hombres y en el lado derecho (Tabla 3).

\section{Asociación con la dehiscencia del canal semicircular superior}

Se han comunicado pacientes con dehiscencia del canal semicircular posterior que también presentan dehiscencia del superior, así, Krombach y $\mathrm{col}^{2}$ describieron 8 pacientes con ambas dehiscencias, Chen y coll1 también observaron un caso, Manzari y Modugno ${ }^{26}$ describen otro caso aislado, Gopen y col 2 casos, Russo y col${ }^{27}$ hallaron 3 pacientes, Saxby y $\mathrm{co}^{21}$ un caso de un niño con dehiscencia bilateral de los canales posteriory unilateral superior, Kundagari y $\mathrm{col}^{28}$ un caso de un paciente con dehiscencia bilateral de los canales semicirculares

Tabla 2. Prevalencia de la dehiscencia del canal semicircular posterior en población infantil

\begin{tabular}{|lcccrr|}
\hline & $\begin{array}{c}\text { Tamaño muestral } \\
\text { (pacientes) }\end{array}$ & $\begin{array}{c}\text { Pacientes con } \\
\text { dehiscencia }\end{array}$ & $\begin{array}{c}\text { Prevalencia } \\
\text { hueso temporal }\end{array}$ & $\begin{array}{c}\text { Grosor } \\
\text { del corte }\end{array}$ & \multicolumn{1}{c|}{ Edad } \\
\hline Saxby et al (2015) & 334 & 7 & $1,2 \%$ & $0,65 \mathrm{~mm}$ & $<18$ años \\
Chen et al (2008) & $138-7$ & 5 & $1,9 \%$ & $0,625 \mathrm{~mm}$ & $3-21$ años \\
Hawigara et al (2012) & 33 & 6 & $20 \%$ & $0,6 \mathrm{~mm}$ & $<2$ años \\
Meiklejohn et al (2015) & 225 & 9 & $16,7 \%$ & $0,5-0,625 \mathrm{~mm}$ & \\
& & 6 & $2,4 \%$ & & $0-6$ meses \\
& 2 & $1,2 \%$ & & $6-11$ meses \\
& 1 & $0 \%$ & & $3-7$ años \\
\hline
\end{tabular}

Tabla 3. Distribución por sexo y lado

\begin{tabular}{|lcrr|}
\hline & Prevalencia & \multicolumn{1}{c|}{ Sexo } & Lado afecto \\
\hline Saxby et al (2015) & $2,1 \%$ & $71,4 \%$ hombres & 5 der vs 3 izq. \\
Meiklejohn et al (2015) & & $50 \%$ hombres & 5 der vs 4 izq. \\
Russo et al (2014) & $1,2 \%$ & $100 \%$ hombres & 3 der vs 2 izq. \\
Erdogan et al (2011) & $1,2 \%$ & $100 \%$ hombres & - \\
Gopen et al (2010) & $100 \%$ & $41,7 \%$ hombres & 11 der vs 2 izq. \\
Chen et al (2008) & $3,8 \%$ & - & 3 der vs 2 izq. \\
\hline
\end{tabular}


posteriores junto con una dehiscencia del canal superior derecho y Dalchow y co $^{29}$ comunicaron un caso de asociación entre ambos canales verticales más una erosión del lateral. Gracia-Tello y col $^{30}$ no encuentran diferencias estadísticamente significativas $(p=0,49)$ en el grosor de los canales posteriores de pacientes que presentaban dehiscencias del superior 2,1 mm (DE: 1,2), en cambio sí que observaron, un grosor disminuido en el canal posterior contralateral a un homónimo dehiscente (3 casos) 1,3 mm (DE: 0,3).

\section{CLÍNICA}

Los pacientes con dehiscencia del canal semicircular posterior pueden ser asintomáticos, presentar clínica auditiva, vestibular o mixta.

La clínica auditiva incluye todos los tipos de hipoacusia (transmisión neurosensorial o mixta) de distintos grados de intensidad, acúfenos (pulsátil 0 no pulsátil), sensación de plenitud ótica, autofonía y amplificación auditiva de sonidos internos del propio paciente (latido del corazón, masticación, movimiento oculares $)^{6,31-35}$.

La clínica vestibular está formada por episodios de vértigo, desequilibrio crónico, fenómeno de Tullio (vértigo, oscilopsias o náuseas inducidas por estímulos muy ruidosos), nistagmus, oscilopsias y signo de Hennebert (vértigo, oscilopsias o náuseas inducidas por cambios de presión en el conducto auditivo externo $27,28,34,36-38$.

Distintos autores intentan describir cuál sería el síntoma predominante en esta patología. Así para Krombach ${ }^{2}$ fueron los vértigos, presentes en el $86 \%$ de los pacientes, para Zhou ${ }^{31}$ fueron la autofonía, presente en el $90 \%$ de los pacientes, para Gopen ${ }^{6}$ fue la hipoacusia de tipo mixta $(67 \%)$ y para Russo ${ }^{27}$ el síntoma más frecuente fue el acúfeno (75\%). De estos estudios se desprende que no existe un síntoma específico.

Hemos encontrado dos estudios que evalúan la presencia de dehiscencias en pacientes asintomáticos, Krombach y col${ }^{2}$ evaluaron a 196 pacientes obteniendo una prevalencia del $0,5 \%$ de pacientes asintomáticos y Erdogan y $\mathrm{col}^{22}$ en 410 pacientes del $1,2 \%$.

Como curiosidad Meehan y col${ }^{39}$ comunican el primer caso de una mujer embarazada con una dehiscencia del canal semicircular posterior que le ocasionaba síntomas vestibulares. Se debe tener en cuenta que la clínica de dehiscencia aparece antes del parto, ya que se podría sospechar que los esfuerzos realizados durante el periodo expulsivo del parto hubieran sido el agente etiológico de la propia dehiscencia.

Stimmer y col $^{40}$ intentan asociar la clínica con el tamaño o la localización de la dehiscencia del canal semicircular posterior sin encontrar una significación estadística según ellos por el escaso número de la muestra.

Russo y col $^{27}$ compararon la clínica de los pacientes con dehiscencia de los canales superior y posterior concluyendo que no existían diferencias significativas entre ambas y Zhou y $\mathrm{col}^{31}$ propusieron que dada la similitud de la clínica de dichas dehiscencias sería más correcto agruparlas en una misma entidad que se denominaría como síndrome de la tercera ventana o síndrome de Minor.

\section{FISIOPATOLOGÍA}

Para explicar la clínica de la dehiscencia del canal semicircular posterior se ha propuesto al igual que en el canal semicircular superior, la teoría de la "tercera ventana" que propone a la dehiscencia del canal como una ventana más del oído interno ${ }^{41}$. La presencia de un orificio en dicho canal hace que disminuya la resistencia del mismo y parte de la energía mecánica procedente de la vibración de la ventana oval se desvía hacia el canal semicircular posterior (fisiológicamente es un circuito hermético donde no llega energía mecánica) produciendo movimientos perilinfáticos, la posterior estimulación de la cresta ampulary dando lugar a los síntomas vestibulares (fenómeno de Tulio, mareos, nistagmus...).

El resto de esa energía mecánica se transmite a través de los movimientos perilinfáticos de las rampas timpánicas y vestibulares de la cóclea al conducto coclear (pero esta energía es menor de la que llegaría fisiológicamente dado que parte de la energía ha ido al canal semicircular) originando una hipoacusia. Gubbels y col${ }^{42}$ proponen que la presencia de acúfeno pulsátil se ha asociado al pulso del golfo de la vena yugular en pacientes con dehiscencias del canal. 


\section{EXÁMENES COMPLEMENTARIOS}

Audiometría tonal liminar: El patrón más frecuente observado en la audiometría de tonos puros de pacientes con dehiscencia del canal semicircular posterior es un gap entre la vía aérea y ósea en los sonidos de baja frecuencia del lado afecto y umbrales por debajo de los $0 \mathrm{~dB}$ de intensidad en la vía ósea ${ }^{31}$. Estos hallazgos fueron corroborados por Nageris $^{43}$ en su estudio experimental con animales.

Prueba de Rinne: En pacientes con dehiscencia del canal semicircular posterior la prueba de Rinne es negativa ${ }^{31}$.

Prueba de Weber: En pacientes con dehiscencia del canal semicircular posterior la prueba de Weber lateraliza al lado afecto ${ }^{31}$.

Reflejo acústico o estapedial: Todos los pacientes valorados por Gopen ${ }^{6}$ y Peress ${ }^{18}$ fueron normales. Sin embargo, Sone ${ }^{44}$ obtuvieron un movimiento ondulatorio en esta prueba.

El reflejo estapedial permite un diagnóstico diferencial entre la hipoacusia de transmisión causada por la dehiscencia del posterior o por una patología del oído medio (fijación de cadena de huesecillos) ya que en la hipoacusia de transmisión originada por alteraciones en oído medio, el reflejo estapedial está ausente mientras que en la causada por la dehiscencia está conservado ${ }^{6}$.

Timpanometría: No muestra alteraciones ${ }^{6-18}$.

Evaluación del nistagmus: El nistagmus en la dehiscencia del canal semicircular posterior es de tipo torsional-vertical ${ }^{45}$.

\section{Prueba de potenciales vestibulares miogénicos} evocados (VEMP): Es una prueba de gran utilidad para el diagnóstico de la dehiscencia del canal semicircular posterior dado que tiene una sensibilidad (91,4\%) y especificidad $(95,8 \%)$ elevadas. El patrón característico es una disminución del umbral de aparición de potenciales inhibitorios con un incremento de su amplitud 6,31,37,46.

Evaluación del reflejo vestíbulo-ocular: El componente vertical del reflejo vestíbulo ocular permitía distinguir si la dehiscencia era del canal semicircular posterior (hacia abajo) o superior (hacia arriba ${ }^{47}$.

\section{Estudios de imagen}

Tomografía computarizada (TC): Es la prueba de imagen de elección para el diagnóstico de la dehiscencia del canal semicircular. Es recomendable realizar, además de reconstrucciones coronales, reconstrucciones en el plano del canal con el fin de minimizar el número de falsos positivos en el diagnóstico. Se han encontrado dos limitaciones en esta prueba; el grosor de corte utilizado en la TC que puede dar un sobrediagnóstico al no permitir identificar capas muy finas de hueso $0^{27}$ y la incapacidad de interpretación del hueso inmaduro en pacientes en edad pediátrica ${ }^{4}$.

Resonancia magnética (RM) también se usa como prueba de imagen. Según Krombach y $\mathrm{col}^{48}$ la RM tiene una sensibilidad del $96 \%$ y una especificidad del $98 \%$ y según Browayes y col$^{49}$ obtuvieron una sensibilidad y un valor predictivo negativo del $100 \%$, una especificidad del $99,1 \%$ y un valor predictivo positivo del $33,3 \%$. Inal y col ${ }^{50}$ concluyen que se realice RM para el diagnóstico de una dehiscencia y solo en caso de duda se debe realizar un examen de TC.

\section{TRATAMIENTO}

El tratamiento inicial en el paciente con dehiscencia del canal semicircular posterior consiste en realizar un seguimiento del mismo recomendándole evitar aquellos estímulos que desencadenan los síntomas. No obstante existen casos donde los síntomas vestibulares 0 auditivos se presentan de manera crónica, o interfieren en la rutina diaria del paciente. En estos casos debe considerarse el tratamiento quirúrgico ${ }^{18}$.

Se han descrito dos vías de abordaje para realizar esta cirugía en función de la localización de la dehiscencia: vía transmastoidea ${ }^{23,42} 0$ mediante craneotomía de la fosa posterior².

Además se han definido dos técnicas en el tratamiento quirúrgico de la dehiscencia del canal semicircular posterior. 
"Plugging": Consiste en rellenar el conducto membranoso del canal semicircular posterior con un tapón de fascia temporal y virutas de hueso desde la ampolla hasta el área dehiscente. Posteriormente se cubre la dehiscencia con pasta de hueso ${ }^{23,51}$.

"Resufacing": Se trata de un cierre de la dehiscencia. Primero se cubre la dehiscencia con un injerto de fascia temporal. Posteriormente se pone una capa de pasta de hueso $0^{37,42}$.

No hemos encontrado ningún trabajo que compare ambas técnicas. Sin embargo Gubbels ${ }^{42}$ justifica la realización de la maniobra "resurfacing" por vía transmastoidea como técnica de elección en dehiscencias asociadas al golfo de la yugular porque esta técnica permite el aislamiento sólido entre el canal semicircular y el golfo yugular.

Es necesario tener presente que siempre que se plantee un tratamiento quirúrgico, se debe valorar los riesgos/beneficios del paciente. Así Peress ${ }^{18}$ no recomienda la cirugía en aquellos pacientes con dehiscencia del canal semicircular cuya indicación quirúrgica sea la hipoacusia porque la corrección de la misma es incierta.

En un esfuerzo por reducir aún más el riesgo que implica la realización de una craneotomía 0 incluso los riesgos del abordaje por la vía transmastoidea se han propuesto modificaciones técnicas

\section{BIBLIOGRAFÍA}

1. Minor LB, Solomon D, Zinreich JS, Zee DS. Soundand/or pressure-induced vertigo due to bone dehiscence of the superior semicircular canal. Arch Otolaryngol Head Neck Surg 1998; 124: 249-58.

2. Krombach GA, Di Martino E, Schmitz-Rode $T$ y coL. Posterior semicircular canal dehiscence: a morphologic cause of vertigo similar to superior semicircular canal dehiscence. Eur Radiol 2003; 13: $1444-50$.

3. Whyte J, Cisneros A, Martínez C y col. Congenital dehiscence in the posterior semicircular canal. Otol Neurotol 2013; 34: 1134-7.

4. Hagiwara M, Shaikh Ja, Fang Y, Fatterpekar G, Roehm PC. Prevalence of radiographic semicircular canal dehiscence in very young children: an evaluation using high-resolution computed tomography of the temporal bones. Pediatr Radio/2012; 42: 1456-64. como es el enfoque transcanal. Esta técnica, basándose en que los síntomas del canal dehiscente son debidos al efecto tercera ventana, sugiere que el cierre o refuerzo de cualquiera de las tres ventanas podrían mejorar o suprimir dichos síntomas. La técnica consiste en reforzar u ocluir las ventanas oval y redonda a través de conducto auditivo externo con una incisión endaura ${ }^{52,53}$. La parte negativa de esta técnica es la transitoriedad en muchos de los casos.

\section{CONCLUSIONES}

La dehiscencia del canal semicircular posterior tiene una etiología mixta admitiéndose en la actualidad un origen congénito 0 adquirido, su prevalencia es variable: $0,3 \%-4,5 \%$ en adultos y $1,2 \%-20 \%$ en niños. Dos son sus localizaciones: golfo de la yugular o fosa cerebral posterior. Los pacientes pueden ser asintomáticos 0 presentar clínica auditiva y/o vestibular. La tomografía computarizada y la prueba de potenciales vestibulares miogénicos evocados permiten establecer el diagnóstico de certeza. El tratamiento quirúrgico se realiza en pacientes con afectación de su rutina diaria. La vía de abordaje de elección es la transmastoidea y se han descrito dos técnicas: "plugging" y "resurfacing".

5. Кoch B, Goold A, Egelhoff J, Benton C. Partial absence of the posterior semicircular canal in Alagille syndrome: CT findings. Pediatr Radiol 2006; 36: 977-9.

6. Gopen Q, Zhou G, Poe D, Kenna M, Jones D. Posterior semicircular canal dehiscence: first reported case series. Otol Neurotol 2010; 31: 339-44.

7. Goddard JC, Oliver ER, Meyer TA. Bilateral posterior semicircular canal dehiscence in the setting of Hallermann-Streiff syndrome. Ear Nose Throat J 2012; 91: 360-3.

8. Kunn JJ, Clenney T. The association between semicircular canal dehiscence and Chiari type I malformation. Arch Otolaryngol Head Neck Surg 2010; 136: 1009-14.

9. Noonan KJ, Russo J, Shen J y col. CDH23 Related hearing loss: A new genetic risk factor for semicircular canal dehiscence? Otol Neurotol 2016; 37 (10): 1583-8. 
10. Cisneros Al, Whyte J, Martínez C y col. Radiological patterns of the posterior semicircular canal. Surg Radiol Anat 2014; 36: 137-40.

11. Chen EY, Paladin A, Phillips G y col. Semicircular canal dehiscence in the pediatric population. Int J Pediatr Otorhinolaryngol 2009; 73: 321-7.

12. Brantberg K, Bagger-Suöbäck D, Mathiesen T, Witt H, Pansell T. Posterior canal dehiscence syndrome caused by an apex cholesteatoma. Otol Neurotol 2006; 27: 531-4.

13. McCall Aa, Curtin HD, McKenna MJ. Posterior semicircular canal dehiscence arising from temporal bone fibrous dysplasia. Otol Neurotol 2010; 31: 1516-7.

14. Kiumehr S, Mahboubi H, Djalilian HR. Posterior semicircular canal dehiscence following endolymphatic sac surgery. Laryngoscope 2012; 122: 2079-81.

15. Cremer PD, Miglaccio AA, Pohl DV y col. Posterior semicircular canal nystagmus is conjugate and its axis is parallel to that of the canal. Neurology 2000; 54: 2016-20.

16. Friedmann DR, Eubig J, Winata LS, Pramanik BK, Merchant SN, Lalwani AK. Prevalence of jugular bulb abnormalities and resultant inner ear dehiscence: an histopathologic and radiologic study. Otolaryngol Head Neck Surg 2012; 147: 750-6.

17. Park JJ, Shen A, Loberg C, Westhofen M. The relationship between jugular bulb position and jugular bulb related inner ear dehiscence: a retrospective analysis. Am J Otolaryngol 2015; 36: 347-5.

18. Peress L. Telian SA. Srinivasan A. Dehiscence of the posterior semicircular canal. Am J Otolaryngol 2015; 36: 77-9.

19. Schutt CA, Kveton JF. Posterior semicircular canal dehiscence secondary to jugular enlargement. Am J Otolaryngol 2016; 37: 173-4.

20. Crovetto MA, Whyte J, Rodriguez 0, Lecumberri I, Martinez C, Eléxpuru J. Anatomo-radiological study of the superior semicircular canal dehiscence radiological considerations of superior and posterior semicircular canals. Eur J Radiol 2010; 76: 167-72.

21. Saxby AJ, Gowdy C, Fandiño M, Chadha NK, Kozak FK ET AL. Radiological prevalence of superior and posterior semicircular canal dehiscence in children. Int J Pediatr Otorhinolaryngol 2015; 79: 411-8.
22. Erdogan N, Songu M, Akay E y col. Posterior semicircular canal dehiscence in asymptomatic ears. Acta Otolaryngol 2011; 131: 4-8.

23. Dang PT, Kennedy TA, Gubbels SP. Simultaneous, unilateral plugging of superior and posterior semicircular canal dehiscences to treat debilitating hyperacusis. J Laryngol Otol 2014; 128: $174-8$.

24. Friedmann DR, Eubig J, Winata LS, Pramanik BK, Merchant SN, Lalwani AK. A clinical and histopathologic study of jugular bulb abnormalities. Arch Otolaryngol Head Neck Surg 2012; 138: 66-71.

25. Nomiya S, Cureoglu S, Kariya S y col. Posterior semicircular canal dehiscence: a histopathologic human temporal bone study. Otol Neuroto/2010; 31: 1122-7.

26. Manzari L, Modugno GC. Bilateral dehiscence of both superior and posterior semicircular canals. Otol Neurotol 2009; 30: 423-5.

27. Russo Je, Crowson MG, DeAngelo EJ, Belden CJ, Saunders JE. Posterior semicircular canal dehiscence: CT prevalence and clinical symptoms. Otol Neurotol 2014; 35: 310-4.

28. Kundaragi NG, Mudali S, Karpagam B, Priya R. Intracranially protruded bilateral posterior and superior SCCs with multiple dehiscences in a patient with positional vertigo: CT and MR imaging findings and review of literature. Indian J Radiol Imaging 2014; 24: 406-9.

29. Dalchow CV, Knecht R, Grzyska U, Muenscher A. Radiographic examination of patients with dehiscence of semicircular canals with digital volume tomography. Eur Arch Otorhinolaryngol 2013; 270: 511-9.

30. Gracia-Tello, Cisneros A, Crovetto R y col. Effect of semicircular canal dehiscence on contralateral canal bone thickness. Acta Otorrinolaringol Esp 2013; 64: 97-101.

31. Zhou G, Gopen Q, Poe DS. Clinical and diagnostic characterization of canal dehiscence syndrome: a great otologic mimicker. Otol Neurotol 2007; 28: 920-6.

32. Paladin AM, Phillips GS, Raske ME, Sie KC. Labyrinthine dehiscence in a child. Pediatr Radiol 2008; 38: 348-50.

33. Friedmann DR, Le BT, Pramanik BK, Lalwani AK. Clinical spectrum of patients with erosion of the inner ear by jugular bulb abnormalities. Laryngoscope 2010; 120: 365-72. 
34. ManzaRI L. Multiple dehiscences of bony labyrinthine capsule.A rare case report and review of the literature. Acta Otorhinolaryngol Ital 2010; 30: 317.

35. Kubota M, Kubo K, Yasui T, Matsumoto N, Komune S. Development of conductive hearing loss due to posterior semicircular canal dehiscence. Auris Nasus Larynx 2015; 42: 245-8.

36. Chien WW, Carey JP, Minor LB. Canal dehiscence. Curr Opin Neurol 2011; 24: 25-31.

37. Lim HW, Park HJ, Jung JH, Chung JW. Surgical treatment of posterior semicircular canal dehiscence syndrome caused by jugular diverticulum. J Laryngol Otol2012; 126: 928-31.

38. Spasic M, Trang A, Chung LK y col. Clinical characteristics of posterior and lateral semicircular canal dehiscence. J Neurol Surg B Skull Base 2015; 76: 421-5.

39. Meehan T, Nogueira C, Rajenderkumar D, Shah J, Stephens D, Dyer K. Dehiscence of the posterior and superior semicircular canal presenting in pregnancy. B-ENT 2013; 9: 165-8.

40. Stimmer H, Hamann KF, Zeiter S, Naumann A, Rummeny EJ. Semicircular canal dehiscence in HR multislice computed tomography: distribution, frequency, and clinical relevance. Eur Arch Otorhinolaryngol 2012; 269: 475-80.

41. Bear ZW, McEvoy TP, MIKULEC AA. Quantification of hearing loss in patients with posterior semicircular canal dehiscence. Acta Otolaryngol 2015; 135: 974- 7.

42. Gubbels SP, Zhang Q, Lenkowski PW, Hansen MR. Ann. Repair of posterior semicircular canal dehiscence from a high jugular bulb. Otol Rhinol Laryngol 2013; 122: 269-72.

43. Nageris Bi, Attias J, Shemesh R, Hadar T, Preis M. A third window of the posterior semicircular canal: an animal model. Laryngoscope 2010; 120: 1034- 7.

44. Sone M, Katayama N, Naganawa $S$, Yoshida $T$, Teranishi M, Nakashima T. Audiological signs in pediatric cases with dehiscence of the bony labyrinth caused by a high jugular bulb. Int $J$ Pediatr Otorhinolaryngol 2012; 76: 447-51.

45. Walther LE, Nath V, Krombach GA, Di Martino E.
Bilateral posterior semicircular canal aplasia and atypical paroxysmal positional vertigo: a case report. Acta Otorhinolaryngol Ital2008; 28: 79-82.

46. Vanspaumen R, Salembier L, Van den Haume L, Parizel P, Wuyts Fl, Van de Heyning PH. Posterior semicircular canal dehiscence: value of VEMP and multidetector CT. B-ENT 2006; 2: 141-5.

47. Aw ST, Aw GE, Todd MJ, Bradshaw AP, Halmagy GM. Three-dimensional vibration-induced vestibulo-ocular reflex identifies vertical semicircular canal dehiscence. J Assoc Res Otolaryngol 2011; 12: 549-58.

48. Krombach GA, Schmitz-Rode T, Haage Pl y col. Semicircular canal dehiscence: comparison of T2-weighted turbo spin-echo MRI and CT. Neuroradiology 2004; 46: 326-31.

49. Browaeys P, Larson TL, Wong ML, Patel U. Can $M R I$ replace $C T$ in evaluating semicircular canal dehiscence? AJNR Am J Neuroradiol 2013; 34: 1421-7.

50. Inal M, Burulday V, Bayar Muluk N y col. Magnetic resonance imaging and computed tomography for diagnosing semicircular canal dehiscence. $J$ Craniomaxillofac Surg 2016; 44 (8): 988-1002.

51. Mikulec AA, Poe DS. Operative management of a posterior semicircular canal dehiscence. Laryngoscope 2006; 116: 375-8.

52. Silverstein H, Kartush JM, Parnes LS y col. Round window reinforcement for superior canal dehiscence. Otolaryngol Head Neck Surg 2012; 147 (2 suppl): 93.

53. Slavutsky V, Nicenboim L. Preliminary results in cochlear implant surgery without antromastoidectomy and with atraumatic electrode insertion: the endomeatal approach. European archives of Otol Rhino Laryngology 2009 4: 481-8.

54. Elmali M, Polat aV, Kucuk H, Atmaca S, Aksoy A. Semicircular canal dehiscence: frequency and distribution on temporal bone CT and its relationship with the clinical outcomes. Eur $J$ Radiol 2013; 82: 606-9.

55. Meiklejohn DA, Corrales CE, Boldt BM y col. Pediatric semicircular canal dehiscence: radiographic and histologic prevalence, with clinical correlation. Otol Neurotol 2015; 36: 1383-9.

\footnotetext{
Dirección: J. Whyte Orozco

Departamento de Anatomía e Histología Humanas, Facultad de Medicina, Universidad de Zaragoza. España Email: jwhyte@unizar.es
} 